\title{
Article
}

\section{Investigation of the use of thermography for research and clinical applications in pregnant women}

\author{
Topalidou, Anastasia and Downe, Soo
}

Available at http://clok.uclan.ac.uk/13979/

Topalidou, Anastasia ORCID: 0000-0003-0280-6801 and Downe, Soo ORCID: 0000-0003-2848-2550 (2016) Investigation of the use of thermography for research and clinical applications in pregnant women. Infrared Physics \& Technology, 75 . pp. 59-64. ISSN 13504495

It is advisable to refer to the publisher's version if you intend to cite from the work. http://dx.doi.org/10.1016/j.infrared.2015.12.016

For more information about UCLan's research in this area go to http://www.uclan.ac.uk/researchgroups/ and search for <name of research Group>.

For information about Research generally at UCLan please go to http://www.uclan.ac.uk/research/

All outputs in CLoK are protected by Intellectual Property Rights law, including Copyright law. Copyright, IPR and Moral Rights for the works on this site are retained by the individual authors and/or other copyright owners. Terms and conditions for use of this material are defined in the policies page.

\section{CLoK}

Central Lancashire online Knowledge www.clok.uclan.ac.uk

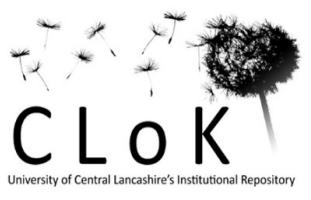




\title{
Investigation of the use of thermography for research and clinical applications in pregnant women
}

\author{
Anastasia Topalidou ${ }^{1}$ and Soo Downe ${ }^{2}$ \\ 1. University of Crete, Faculty of Medicine, Department of Orthopaedics and \\ Traumatology \\ 2. University of Central Lancashire, School of Health
}

\begin{abstract}
Background: The possibility of using thermal imaging, as a non-invasive method, in medicine may provide potential ability of advanced imaging.

Objective: The conduction of a preliminary study in healthy non-pregnant females in order to investigate the imaging ability of thermography and its implementation; and to determine hot and cold areas in order to create a "map" of temperature distribution of the abdomen and the torso.
\end{abstract}

Methods: Participants were 18-45 years old non-pregnant women $(n=10)$, who were measured at 4 different distances. Two thermal imaging cameras and their corresponding software were used to measure abdomen, low back, left and right side of the torso.

Results: There were no statistically significant differences in the mean values of the exported temperatures according the distance and the angle between the camera and the subject. The inferior part of the rectus abdominis muscle recorded the coldest zone and the umbilicus appeared as the most prominent hot spot.

Conclusions: Thermography shows to be a potential non-invasive technique offering new options in the evaluation of pregnant and laboring women.

Keywords: thermography, infrared, pregnancy, region of interest, abdomen

\section{Introduction}

Many studies have been conducted in order to better understand the nature and consequences of fetal and maternal movement during pregnancy and childbirth. These include analyses of the function and structure of the body of the pregnant woman, and of the fetus [1-7]. Research in this area has, however, very rarely examined the dynamic interaction of the mother and the fetus at the biomechanical level, especially during the process of labour. This is despite the fact that there is good quality evidence from applied studies that upright positions and maternal movement during labour are associated with positive outcomes for 
mother and for baby. One of the barriers to such research has been the lack of appropriate, non-invasive, safe imaging techniques for visualizing the position and movements of the fetus in relation to those of the mother during the process of labour.

Thermography (digital infrared thermal imaging) is a non-invasive method with the ability of real-time monitoring and imaging, which has been used in medicine since the early 1960's. It uses no radiation and no contact, is free from any limitations or contra-indications, is easy to use, and provides objective data. To date, the method has been introduced in the clinical research area for diagnosis, and for the prevention of various diseases [8-13]. However, to the best of our knowledge, apart from some applications of the method in the evaluation of preterm premature rupture of the fetal membranes [14], chorioamnionitis [15] and peripheral temperature of abdominal wall $[15,16]$, there are no scientific studies to investigate the use of thermography in pregnant women. The possibility of using this method in pregnant women may provide advanced imaging of fetal movements and the potential ability to record the movements of the mother and the fetus, simultaneously, to see how they interact and interrelate as the fetus negotiates the pelvis, in the process of being born.

The purpose of this research was to undertake a preliminary study in healthy nonpregnant female volunteers in order to investigate the imaging ability of thermography and its implementation in relation to the female abdomen and torso; to determine hot and cold areas; and to create a "map" of temperature distribution in this field, as a basis for future modeling and empirical studies that can be applied to pregnancy and labour.

\section{Participants and Methods}

2.1 Participants: 10 healthy non-pregnant female volunteers aged $18-45$ years took part. All participants were provided with a participant's information sheet, informing them in detail about the purpose of and the protocol for the study, and all gave written consent. Exclusion criteria and participants' protocol are presented in Table 1 . The study was conducted exclusively in women, as it is the first stage in developing a flexible, non-invasive technique for assessing maternal/fetal movement during pregnancy and birth, and as intended for further use in pregnant and due to uniformity of the sample, and as it is known that the difference in the anatomy of human body between women and men and the different temperature emitted by vessels and organs within the body, results in the export of different temperatures from the same anatomical sites $[17,18]$.

2.2 Technique and measurement procedure: After acclimatization, all measurements were conducted in the same laboratory environment at a constant temperature of $24,5^{\circ}-25,5^{\circ} \mathrm{C}$ $[19,20]$. Only the research team and the participant were in the room there the measurements took place, ensuring a consistent number of people in the room, to avoid the influence of human body at room temperature. On the arrival at the lab, participants were asked to remove all their jewelry and the clothes in the area of measurement (abdomen/back). Then they were asked to stand for 15 minutes with the abdomen/back exposed to achieve skin temperature equilibration with the temperature of the room $[21,22]$. 
The image processing equipment and other devices were sited away from the participants' location, to avoid heat disturbance [23]. For the measurement procedure two thermal imaging cameras (ThermoVision A40M and RayCAm C.A. 1884) and their corresponding software were used. ThermoVision A40M was mounted on a tripod height 1.2 meters and RayCAm C.A. 1884 was carried by an examiner, at the same height as the other camera, having the hand stabilized in a base. The distance between the cameras and the participant were identified by the lens which is integral to the camera. The cameras' lenses were parallel to the area of measurement. Human skin emissivity was set at 0.97-0.98 [23].

The types of measurements were as follows:

a) All participants were measured at 4 different distances from the cameras $(5 \mathrm{~m}, 4 \mathrm{~m}$, $3 \mathrm{~m}$ and $2 \mathrm{~m}$ ). They were asked to stand still in an anatomical position at defined points, facing the camera, for as long as the region of interest (RI) was recorded. Examinees were asked to place their index fingers at the Anterior Superior lliac Spine (ASIS), to define two reference points for analysis reasons.

b) At the last point $(2 \mathrm{~m}), 4$ measurements were performed by a rotation around the vertical axis (abdomen, back, left and right side of the torso) (Figure 1).

The Ethics Committee of University of Central Lancashire, UK, approved this study.

2.3 Statistical analysis: Repeated measures analyses of variance (ANOVA) with Bonferroni adjustment were used to test whether there was a significant difference in temperature recordings at 5, 4, 3 and $2 \mathrm{~m}$. It was also used to compare the recordings from the different angles (abdomen, back, left and right side) in a $2 \mathrm{~m}$ distance between the camera and the body. All statistical tests were carried at the $5 \%$ level of significance.

In the first study, the null hypothesis was that the recorded temperature would be the same in each measurement, and that this wound not depends on the distance between the camera and the examinee. If the null hypothesis was rejected then this was assumed to be strong indication that distance was a main factor in the recorded data.

In the second study, the null hypothesis was that the temperature recording would be the same in each measurement and this would not depend on the camera's angle. If the null hypothesis was rejected then this was assumed to be strong indication that the camera's angle was an important factor in the recorded data. SPPS 15.0 was used for statistical analysis.

\section{Results}

The demographic characteristics of the participants are presented in Table 2. No statistically significant differences were exhibited in exported temperatures of the two cameras. The mean values (MV) and the standard deviation (SD) of temperatures recorded according the distance and the angle between the camera and the subject, the statistically significant differences $(p<0.005)$ of all measurements, and the differences that occurred during analysis of the whole image (WI) compared to the RI are presented in Table 3. 
In the first study, the repeated measures ANOVA revealed that there was no statistically significant difference in temperature recording in the RI (Table 4) when the distance between the subject and the camera differed. From the graphical representation of the above results (Figure 2) it is clear that regardless of the point at which the examinee or any area of study is sited, the observed values are identical, with only some minor differences in their standard deviations.

In the second study, the repeated measures ANOVA revealed that there was no statistically significant difference in temperature recording in the RI (Table 5) when the angle between the participant and the camera differed, regardless of which side of the body was recorded (abdomen, back, left or right side).

The corresponding infrared thermograms with points displaying maximum and minimum values for both $\mathrm{WI}$ and $\mathrm{RI}$ are depicted in Figure 3. Figure 4 shows the isotherm analysis of the abdomen.

\section{Discussion}

To our knowledge, this is the first study examining the abdomen, the back and the lateral regions of the trunk in female participants, using thermography with the main aim of investigating the imaging capabilities of the method and its extracts, as a non-invasive method without radiation, with the view to future use and application with pregnant and laboring women.

The individuals who participated in this study had a normal BMI average. This could be a limitation to extension of the results to whole populations of women, as it is known that in people with normal BMI, less thermal contours are recorded in the torso region, compared with overweight and obese individuals, suggesting a narrower temperature in the study area [24]. The small sample size is also a limitation, but the consistency of the findings between the participants suggests that this is unlikely to have significantly biased the results.

If an imaging technique is to be used in labour, it needs to be flexible enough to cope with shifting degrees of distance from the camera, as the laboring woman changes position spontaneously in response to the movements of her fetus. The lack of statistically significant differences between the measurements at various differences is therefore reassuring (Fig 2). However, while the measurements were made at four different distances [25], the image captured at 2 meters generate the possibility of a more detailed image processing, although a 3 meter distance also resulted in satisfactory resolution with good processability. This means that the distance does not play a role with respect to the exported data but, in case of image processing the distance of 2 and 3 meters gives a better image resolution.

On the assumption that in pregnant and/or laboring women the study area is larger, and more similar as to the area and curvature, as those presented be overweight individuals $[23,24]$, recording at a slightly larger distance than 2 meters, only in case of image processing and not regarding data export, is recommended. 
Similar to the study of Heuberger et al (2012) [24], in our study in all subjects and in all measurements the inferior part of the rectus abdominis muscle recorded the coldest zone. In the RI the umbilicus appeared as the most prominent hot spot: so much so that it be used as a reference point (Fig. 4- Left Image). The very evident temperature variation at this point of RI may be due to abdominal fatty deposits. It has been shown that as the body fat increases there is a greater variation in temperature distribution of the body [18]. The region with the lowest temperature in the current study is that which would contain the fetus during pregnancy. This is an important finding, as further study in pregnant women may be able to highlight thermal variation in this area that could provide an image of the fetus that is distinct from that of the mother. The amniotic fluid and the temperature of the body of the fetus may offer diversity in the representation of the temperatures. The mapping of this area and the possibility of fetal representation in correlation with another imaging method could provide the basis for a future model of non-invasive recording of the position of the fetus in dynamic interaction with mother.

Moreover, based on the results the distance from the camera and the angle between the body and the camera did not influence the thermal imaging capacity of the method. This indicates that thermography is an objective method, which has both the ability to record and display the abdomen and the back, throughout the surrounding region, providing invariable results, regardless of the point or position the participant adopt. This means that a thermal record of the body of an individual who moves or rotates can yield satisfactory and reliable results. Moreover, it is important to define the RI in each measurement [23], because as the Table 3 shows, apart from the maximum temperature which is recorded on the body, the minimum temperature and the mean value differ statistically significant when the $W I$ is comparing with the RI, only. This is because in the first case (WI), the minimum temperatures are recorded outside from the body, in the surrounding objects.

\section{Conclusion}

Thermography appears to be an objective and reliable method for imaging the female abdomen and lower back region, as there were no differences in the exported values of the two cameras used in this study. Also, the distance and the angle between body and camera were not important factors for method's performance. The optimum distance for image analysis of the abdomen and torso seemed to be around 2 meters, but greater or lesser distances did not lead to statistically significant variation regarding the exported data. To export objective temperature values, the definition and the delineating of the RI is necessary, in order to exclude the recording of temperature values outside the body. Finally, the area of the abdomen shows a clear thermal gradient, stable in all participants regardless of distance and other characteristics. Thermography therefore seems to be a promising alternative noninvasive technique offering new options in future studies in pregnant and laboring women, providing useful information which cannot be easily or safely obtained with existing techniques. Future studies in pregnant women should be conducted to extract more specific conclusions. 


\section{Acknowledgements}

The author will like to thank Professor Jim Richards, Professor James Selfe, researcher Jill Alexander and Dr George Tzagarakis for assisting in the conduction of the study and the assignment of the laboratory. This paper is part of the COST Action IS0907, "Childbirth Cultures, Concerns and Consequences:Creating a dynamic EU framework for optimal maternity care". The Action aimed to advance scientific knowledge about ways of providing optimum maternity care provision and outcomes for mothers, babies and families across Europe.

\section{References}

1. Berg A. Childhood neurological morbidity and its association with gestational age, intrauterine growth retardation and perinatal stress. Paediatric and Perinatal Epidemiology 1998; 2(3):229-239. [DOI: 10.1111/j.1365-3016.1988.tb00213.x]

2. Lu GC, Rouse DJ, DuBard M, Cliver S, Kimberlin D, Hauth JC. The effect of the increasing prevalence of maternal obesity on perinatal morbidity. Am J Obstet Gynecol 2001; 185(4):845-849. [DOI: http://dx.doi.org/10.1067/mob.2001.117351]

3. Osman NAABU and Ghazali RMAT. Biomechanical evaluation on gait pattern of pregnant subjects. Journal of Mechanics in Medicine and Biology 2002; 2(1):99116. [DOI: $10.1142 /$ S0219519402000289]

4. Gilleard W, Crosbie J, Smith, R. Effect of pregnancy on trunk range of motion when sitting and standing. Acta Obstet Et Gynecol Scand 2002; 81:1011-1020. [DOI: $10.1034 /$ j.1600-0412.2002.811104.x]

5. Sameni $R$ and Clifford GD. A review of fetal ECG signal processing; Issues and promising directions. The Open Pacing, Electrophysiology and Therapy Journal 2010; 3:4-20. [DOI: 10.2174/1876536X01003010004]

6. Forczek $W$ and Staszkiewicz R. Changes of kinematic gait parameters due to pregnancy. Acta Bioeng Biomech 2012; 14(4):113-119. [DOI: 10.5277/abb120413]

7. Gilleard WL. Trunk motion and gait characteristics of pregnant women when walking: report of a longitudinal study with a control group. BMC Pregnancy Childbirth 2013; 13:71-78. [DOI:10.1186/1471-2393-13-71]

8. Meknas K, Odden-Miland A, Mercer JB, Castillejo M, Johansen O. Rediofrequency microtenotomy: a promising method for treatment of recalcitrant lateral epicondylitis. Am J Sports Med 2008; 36(10):1960-1965. [ DOI: 10.1177/0363546508318045] 
9. Kopsa H, Czech W, Schmidt P, Zazgornik J, Pils P, Balcke P. Use of thermography in kidney transplantation: two year follow up study in 75 cases. Proc Eur Dial Transplant Assoc 1979; 16:383-387.

10. Holmgren $\mathrm{K}$, Jacobsson $\mathrm{H}$, Johnsson $\mathrm{H}$, Löfsjögård-Nilsson $\mathrm{E}$. Thermography and plethysmography, a non-invasive alternative to venography in the diagnosis of deep vein thrombosis. J Intern Med 1990; 228(1):29-33. [DOI: 10.1111/j.13652796.1990.tb00188.x]

11. Kennedy DA, Lee T, Seely D. A comparative review of thermography as a breast cancer screening technique. Integr Cancer Ther 2009; 8(1):9-16.

[DOI: 10.1177/1534735408326171]

12. Hildebrandt C, Raschner C, Ammer K. An Overview of Recent Application of Medical Infrared Thermography in Sports Medicine in Austria. Sensors 2010; 10(5):4700-4715. [ DOI: 10.3390/s100504700]

13. Klamann MK, Maier AK, Gonnermann J, Klein JP, Pleyer U. Measurement of dynamic ocular surface temperature in healthy subjects using a new thermography device. Curr Eye Res 2012; 37(8):678-683. [DOI: 10.3109/02713683.2012.674610]

14. Sheinberg M, Hayashi R, Bromley J, Dormer L. Application of telethermography in the evaluation of preterm premature rupture of the fetal membranes. Biomed Instrum Technol 1996; 30(6):526-530.

15. Goodlin RC and Brooks PG. Abdominal wall hot spots in pregnant women. J Reprod Med 1987; 32(3):177-180.

16. Beinder $E$, Huch A, Huch R. Peripheral skin temperature and microcirculatory reactivity during pregnancy. A study with thermography. J Perinat Med 1990; 18(5):383-390. [DOI: 10.1515/jpme.1990.18.5.383]

17. Dębiec-Bąk A., Skrzek A. The comparison of surface body temperature distribution between men and women by means of thermovision. Inż. Biomed. 2012; 18: 2529.

18. Domina T, Kinnicutt $P$, MacGillivray $M$. Thermal pattern variations analyzed using 2D/3D mapping techniques among females. Journal of Textile and Apparel, Technology and Management, 2011; 7(1):1-15.

19. Ishigaki $T$, Ikeda $M$, Asai $H$, Sakuma S. Forehead-Back thermal ratio for the interpretation of infrared imaging of spinal cord lesions and other neurological disorders. Thermology 1989; 3:101-117.

20. Mabuchi K, Kanbara O, Genno H, Chinzei T, Haeno S, Kunimoto M. Automatic control of optimum ambient thermal conditions using feedback of skin temperature. Biomedical Thermology 1997; 16:6-13.

21. Ring EFJ. Computerized thermography for osteoarticular diseases. Acta thermographica 1976; 1:166-173.

22. Roberts DL, Goodman PH. Dynamic thermoregulation of back and upper extremity by computer-aided infrared imaging. Thermology 1987; 2:573-577.

23. Ring EFJ, Ammer K. The technique of infrared imaging in medicine. Thermology Int 2000; 10:7-14. 
24. Heuberger $R$, Kinnicutt $P$, Domina $T$. The relationship between thermal imaging and waist circumference in young adults. Health 2012; 4(12A):1485-1491. [DOI: 10.4236/health.2012.412A213]

25. Ring EFJ and Ammer K. Infrared thermal imaging in medicine. Physiol Meas 2012; 33:R33-R46. [DOI: 10.1088/0967-3334/33/3/R33] 


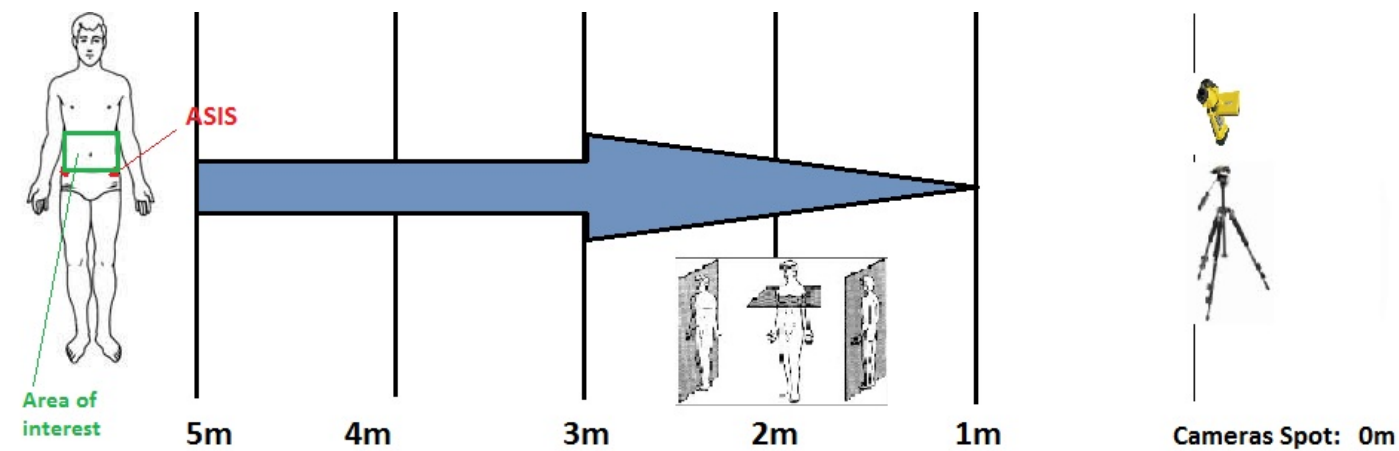

Figure 1. Graphical representation of the measurement procedure 

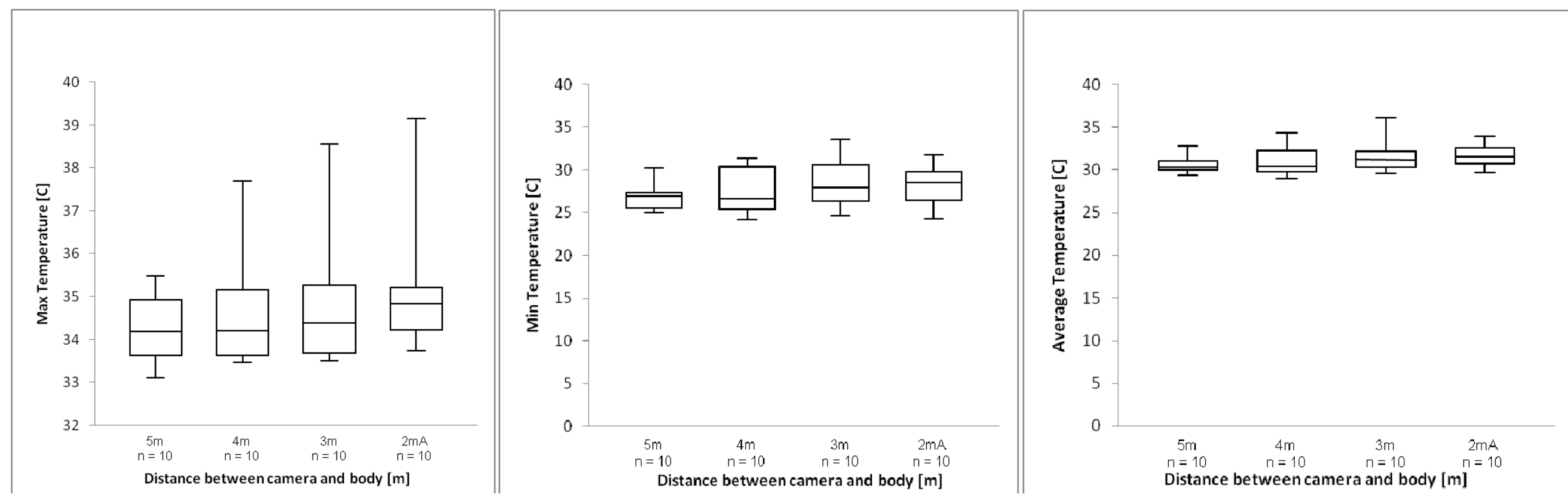

Figure 2. Box plot representation of the temperature's measurements (average temperature) when the distance between the body and camera differs. 
Figure 3. Infrared thermograms of all positions which measured with maximum and minimum temperature values.

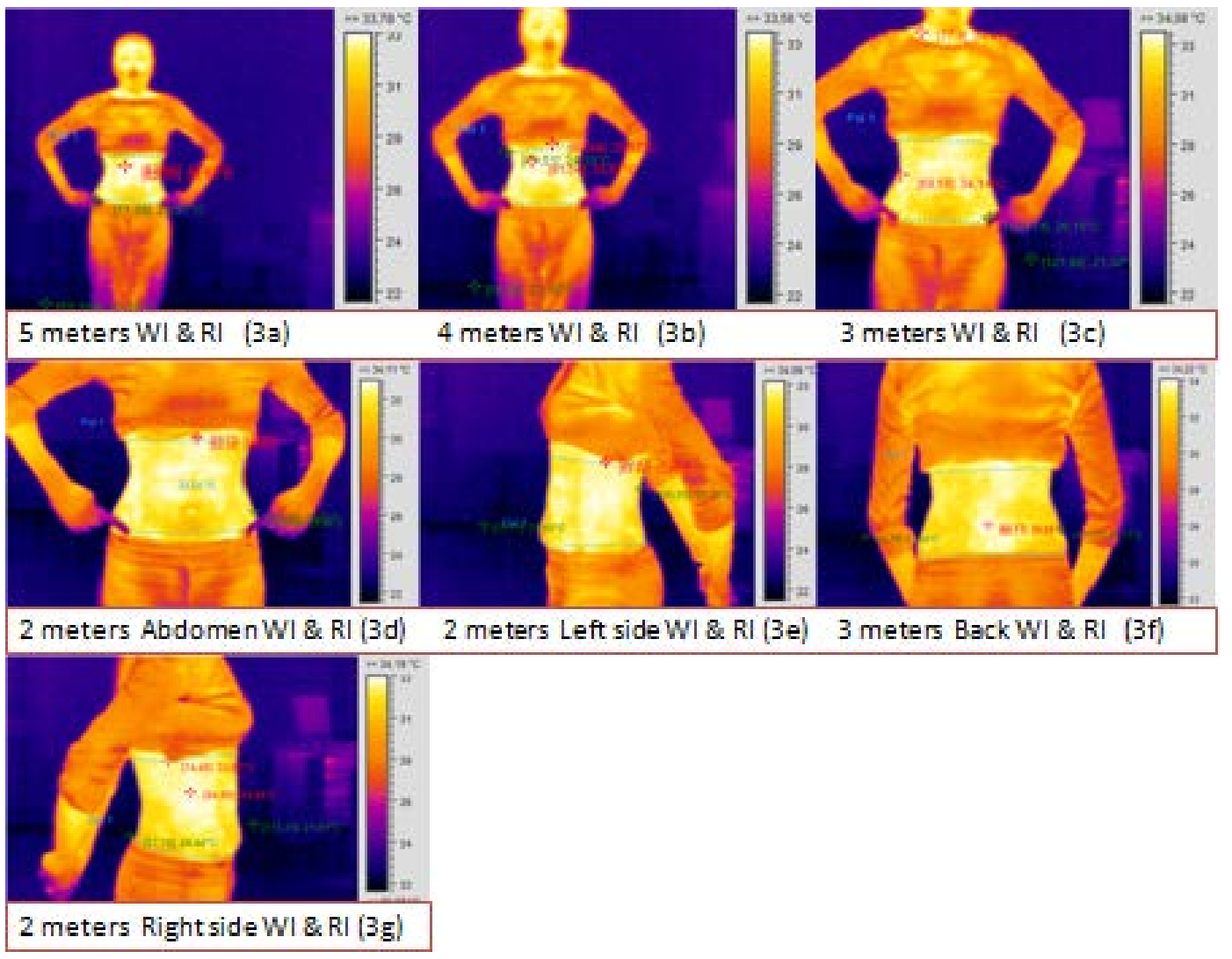


Figure 4. Left image: The area with the lowest temperature. Right image: Isotherm analysis of the abdomen. The red area within field displays an average value of $32,98^{\circ} \mathrm{C}$. The warmest point is umbilicus.

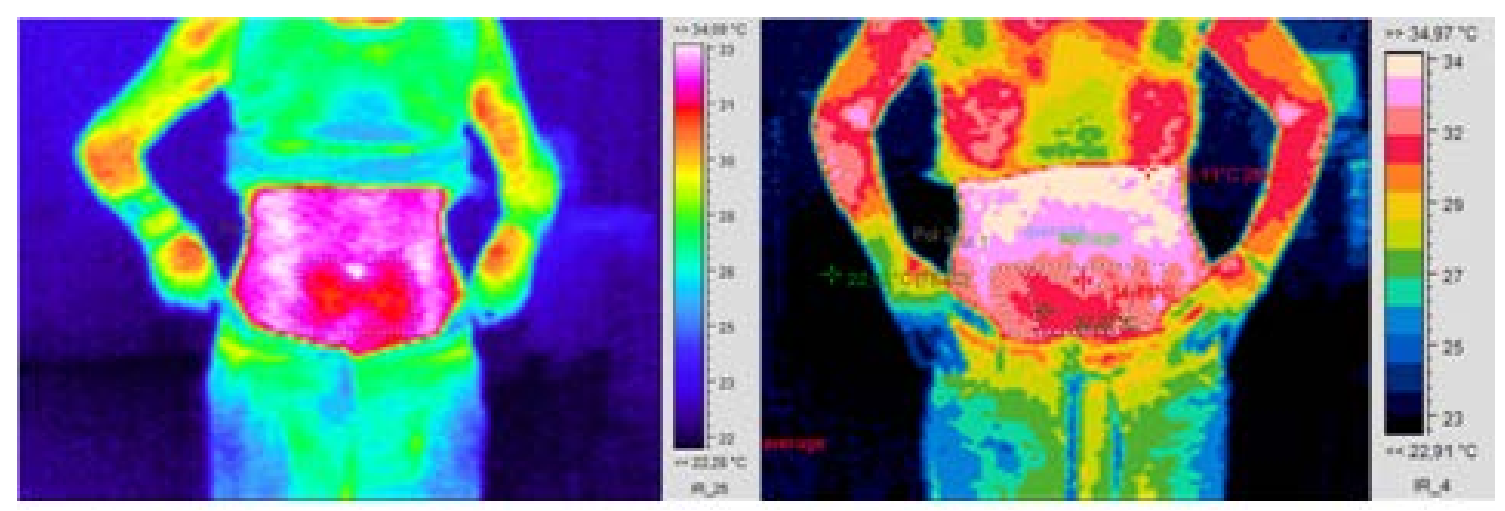


Table 1. Exclusion criteria and participants' protocol

Exclusion Criteria

Participants' protocol

\begin{tabular}{|c|c|}
\hline $\begin{array}{l}\text { Any medication affecting the cardiovascular } \\
\text { system }\end{array}$ & $\begin{array}{l}\text { No ointments or cosmetics on the RI on the } \\
\text { day of exam }\end{array}$ \\
\hline Pain medication at the day of exam & $\begin{array}{l}\text { No alcohol intake or smoking } 24 \text { hours } \\
\text { before the exam }\end{array}$ \\
\hline Solarium or sunbathing 5 days before exam & $\begin{array}{l}\text { Not allowed tight fitting clothing and } \\
\text { shaving of the RI } 4 \text { hours prior the exam }\end{array}$ \\
\hline $\begin{array}{l}\text { Any pathology or inflammations in the } \\
\text { region of interest (RI) }\end{array}$ & $\begin{array}{l}\text { No physical therapy and exercise } 24 \text { hours } \\
\text { before exam }\end{array}$ \\
\hline Medical implants in the body in the RI & $\begin{array}{l}\text { No hot or cold packs application } 24 \text { hours } \\
\text { before the exam }\end{array}$ \\
\hline Women during menstruation & $\begin{array}{l}\text { Bathing or shower no closer than } 1 \text { hour } \\
\text { before exam }\end{array}$ \\
\hline
\end{tabular}


Table 2. Demographic characteristics (Participants: females $n=10$ )

\begin{tabular}{|c|c|c|c|}
\hline & Mean value \& SD & & Mean value \& SD \\
\hline \multirow{2}{*}{$\begin{array}{l}\text { Age (years) } \\
\text { Weight } \\
\text { (kg) }\end{array}$} & $35,8 \quad( \pm 8,19)$ & Height (m) & $1,67 \quad( \pm 0,08)$ \\
\hline & $63,69( \pm 9,21)$ & BMI & $22,94( \pm 3,84)$ \\
\hline \multirow[t]{2}{*}{ Ethnicity } & \multicolumn{3}{|l|}{ Caucasian $\mathrm{n}=7$} \\
\hline & \multicolumn{3}{|l|}{ Asian $\quad \mathrm{n}=3$} \\
\hline \multirow[t]{2}{*}{ Exercise } & Yes & \multirow{2}{*}{$\begin{array}{l}\text { Once a week } \\
4-5 \text { times/week }\end{array}$} & $2-3$ times/week $n=6$ \\
\hline & $\mathrm{n}=1$ & & 6-7 times/week $n=0$ \\
\hline \multirow[t]{2}{*}{ Smoking } & $\mathrm{n}=1$ & \multirow{2}{*}{\multicolumn{2}{|c|}{$<1$ pack/day }} \\
\hline & $n=9$ & & \\
\hline \multirow[t]{2}{*}{ Alcohol } & $n=9$ & $<1$ drink/week & 1-4 drinks/week $n=4$ \\
\hline & $\mathrm{n}=1$ & 5-10 drinks/week $n=3$ & \\
\hline
\end{tabular}


Table 3. The MV, the SD and the statistical significant differences of all measurements.

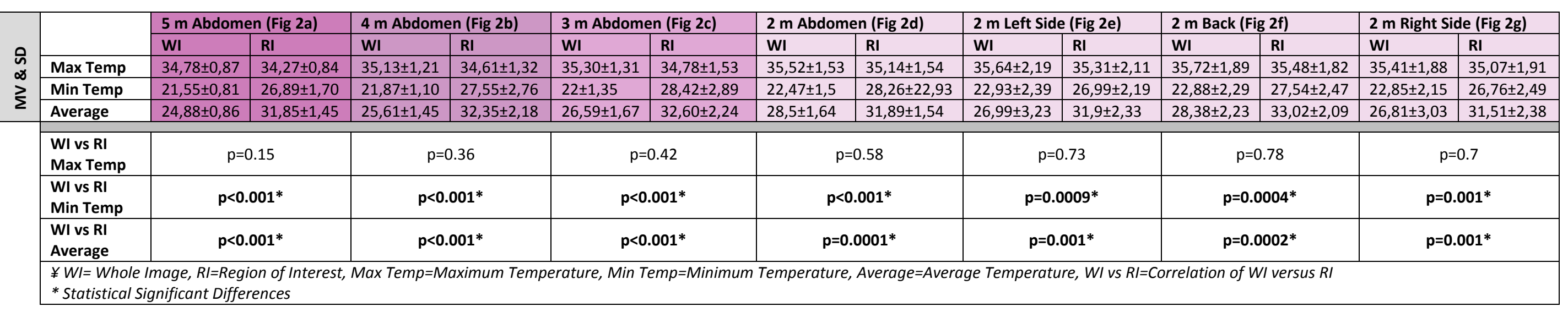


Table 4. Anova's results from based on temperature comparison by differentiating the distance between the camera and the subject.

\begin{tabular}{|c|c|c|c|c|c|}
\hline & \multicolumn{5}{|c|}{ Pairwise comparison } \\
\hline \multirow{4}{*}{$\begin{array}{c}\text { Max } \\
\text { Temp }\end{array}$} & Distance [m] & Distance [m] & Sig. ${ }^{*}$ & Lower bound & Upper bound \\
\cline { 2 - 6 } & 5 & 4 & 1,000 & $-1,121$ & 0,443 \\
\cline { 2 - 6 } & 5 & 3 & 0,670 & $-1,489$ & 0,465 \\
\cline { 2 - 6 } & 5 & 2 & 0,175 & $-2,004$ & 0,260 \\
\cline { 2 - 6 } & 4 & 3 & 1,000 &,- 624 & 0,278 \\
\cline { 2 - 6 } & 3 & 2 & 0,091 & $-1,133$ & 0,067 \\
\hline Min & 5 & 2 & 0,118 &,- 788 & 0,068 \\
\cline { 2 - 6 } & 5 & 3 & 1,000 & $-3,264$ & 1,940 \\
\cline { 2 - 6 } & 5 & 2 & 0,831 & $-4,694$ & 1,636 \\
\cline { 2 - 6 } & 4 & 3 & 0,989 & $-2,796$ & 0,773 \\
\cline { 2 - 6 } & 4 & 2 & 1,000 & $-3,144$ & 1,062 \\
\cline { 2 - 6 } & 3 & 2 & 1,000 & $-2,124$ & 2,442 \\
\hline Average & 1 & 2 & 1,000 & $-2,050$ & 1,049 \\
\cline { 2 - 6 } & 1 & 3 & 0,656 & $-2,952$ & 0,911 \\
\cline { 2 - 6 } & 1 & 4 & 0,108 & $-2,427$ & 0,185 \\
\cline { 2 - 6 } & 2 & 3 & 0,870 & $-1,616$ & 0,576 \\
\cline { 2 - 6 } & 2 & 4 & 0,829 & $-1,904$ & 0,663 \\
\cline { 2 - 6 } & 3 & 4 & 1,000 & $-1,323$ & 1,122 \\
\hline
\end{tabular}

*Adjustment for multiple comparisons: Bonferroni 
Table 5. Anova's results from based on temperature comparison by differentiating the angle between the camera and subject (side of the body).

\begin{tabular}{|c|c|c|c|c|c|}
\hline & \multicolumn{5}{|c|}{ Pairwise comparison } \\
\hline \multirow{4}{*}{$\begin{array}{c}\text { Max } \\
\text { Temp }\end{array}$} & Angle & Angle & Sig. & Lower bound & Upper bound \\
\cline { 2 - 6 } & Abdomen & Left & 1,000 & $-1,053$ & 0,707 \\
\cline { 2 - 6 } & Abdomen & Back & 0,930 & $-1,090$ & 0,402 \\
\cline { 2 - 6 } & Abdomen & Right & 1,000 & $-0,847$ & 0,981 \\
\cline { 2 - 6 } & Left & Back & 1,000 & $-0,814$ & 0,472 \\
\cline { 2 - 6 } & Left & Right & 1,000 & $-0,561$ & 1,041 \\
\cline { 2 - 6 } & Back & Right & 0,017 & 0,071 & 0,751 \\
\hline Min & Abdomen & Left & 1,000 & $-1,742$ & 4,274 \\
\cline { 2 - 6 } & Abdomen & Back & 1,000 & $-3,499$ & 4,931 \\
\cline { 2 - 6 } & Abdomen & Right & 0,405 & $-0,926$ & 3,916 \\
\cline { 2 - 6 } & Left & Back & 1,000 & $-2,892$ & 1,792 \\
\cline { 2 - 6 } & Left & Right & 1,000 & $-1,458$ & 1,916 \\
\cline { 2 - 6 } & Back & Right & 1,000 & $-2,349$ & 3,907 \\
\hline Average & Abdomen & Left & 1,000 & $-1,208$ & 2,301 \\
\cline { 2 - 6 } & Abdomen & Back & 1,000 & $-2,032$ & 2,404 \\
\cline { 2 - 6 } & Abdomen & Right & 0,480 & $-0,551$ & 2,113 \\
\cline { 2 - 6 } & Left & Back & 1,000 & $-1,520$ & 0,799 \\
\cline { 2 - 6 } & Left & Right & 1,000 & $-0,799$ & 1,268 \\
\cline { 2 - 6 } & Back & Right & 1,000 & $-1,019$ & 2,209 \\
\hline
\end{tabular}

*Adjustment for multiple comparisons: Bonferroni 